\title{
Expanding anaplastic lymphoma kinase therapeutic indication to early stage non-small cell lung cancer
}

\author{
Fabrizio Tabbò, Silvia Novello \\ Department of Oncology, University of Torino, Torino, Italy \\ Contributions: (I) Conception and design: All authors; (II) Administrative support: All authors; (III) Provision of study materials or patients: All \\ authors; (IV) Collection and assembly of data: All authors; (V) Data analysis and interpretation: All authors; (VI) Manuscript writing: All authors; (VII) \\ Final approval of manuscript: All authors. \\ Correspondence to: Fabrizio Tabbò. Department of Oncology, University of Torino, Torino, Italy. Email: fabrizio.tabbo@unito.it.
}

\begin{abstract}
Oncogene-addicted non-small cell lung cancer (NSCLC) patients have been witnessing overwhelming therapeutic improvements, especially in advanced disease management, due to the advent of more potent tyrosine kinase inhibitors (TKIs). However, the concrete chance to cure anaplastic lymphoma kinase (ALK)-rearranged patients remains prerogative of surgical and peri-operative medical approaches to early disease stage. Clinical investigations in the adjuvant setting of genotype-restricted lung cancers (i.e., EGFR+, ALK+, etc.) are under-represented owing to the need of large patients' enrollment and solid overall survival (OS) data, which solely can show real long-term impact of these therapeutic interventions. Current available radiological and molecular technologies will widely increase the number of surgical early stage patients, including ALK+, spurring the development of rational approaches aimed to prevent disease recurrence and prolong patients' survival. Ongoing clinical trials, evaluating crizotinib and alectinib as adjuvant treatments, will gauge the real impact of 'TKIs in terms of patients' disease free survival (DFS) and OS; other peri-operative investigations (e.g., neo-adjuvant strategies) will add information about ALK inhibitors' tumor growth restraint capacities and early adaptation mechanisms to ALK targeting. Nevertheless, multiple questions are and will remain unanswered: if should be treated indifferently all ALK+ patients or, alternatively, should be stratified in different risk groups based on the detectable residual disease [i.e., minimal residual disease (MRD) after surgery]; whether ALK inhibitors administration could facilitate the accumulations of persister cells driving resistance mechanisms to targeted therapies; if alternative strategies, as combined treatments targeting different molecular hubs, could enhance disease control and cancer eradication.
\end{abstract}

Keywords: Anaplastic lymphoma kinase (ALK); adjuvant therapy; non-small cell lung cancer (NSCLC); tyrosine kinase inhibitor (TKI); minimal residual disease (MRD)

Submitted Mar 26, 2019. Accepted for publication Jul 15, 2019.

doi: $10.21037 /$ tlcr.2019.07.07

View this article at: http://dx.doi.org/10.21037/tlcr.2019.07.07

\section{Introduction}

Anaplastic lymphoma kinase (ALK) targeting has been, undoubtedly, one of the most revolutionized fields among oncogene-addicted lung cancer patients. In the recent years we can number clinical trials, such as phase 3 randomized ALEX and ALTA-1L, which represent huge milestones in the therapeutic management of advanced ALK+ non- small cell lung cancer (NSCLC) patients $(1,2)$. Both these trials evaluated the activity of a second-generation tyrosine kinase inhibitor (TKI), respectively alectinib for ALEX and brigatinib for ALTA-1L, compared to first-generation TKI, crizotinib, the gold standard therapeutic choice for these patients (1-3). Indeed, more potent ALK inhibitors (ALKi) have been showing deeper and more durable disease control, leading to results hardly imaginable before 
[i.e., median progression-free survival (PFS) of 34.8 months for patients receiving alectinib versus 10.9 crizotinib] (4). Based on these data, alectinib has been approved as first-line treatment option for ALK+ NSCLC patients and, likely, other actors will play major role in the upfront management of these patients.

Despite these improvements in the advanced setting, a thick fog of uncertainty wraps around the role of ALK-targeting in early/locally advance stage of the disease and whether ALKi play any role in this scenario still remains vague. Therefore, ALK rearrangements are not routinely screened in patients' specimens at these disease stages, unless required when enrolled within specific clinical trials (5).

In early stages NSCLC, surgery still represents the potentially curative approach, aimed to eliminating all the radiological detectable tumor bulk. However, a large fraction of patients, roughly $50 \%$, relapse with single or multiple metastases and die for lung cancer (5-year survival rate equal to $56 \%$ ) (6). Having in mind these poor outcomes and lung cancer screening implementation in the very nextfuture, which will increase fraction of surgical patients, is easy to gauge the urgent need of peri-operative (adjuvant and neo-adjuvant) treatment strategies capable to really impact on long-term patients' survival benefit (7-9). That said, current standard approach in the adjuvant setting is still represented by cisplatin-doublet chemotherapy, based on a survival benefit of $5.4 \%$ at 5 years follow up (10).

Genomic complexity of lung adenocarcinoma is well characterized, supporting the evidence of different tumor entities with different biology, natural history and clinical behavior (11). Within oncogene-addicted tumors itself, fusions-positive lung cancers retain peculiar aspects, compared to mutation-driven cancers (e.g., EGFR+) $(12,13)$. Thus, a deep understating of ALK fusionsrelated clinical implications in early stage of the disease is mandatory, to precisely determine their prognostic relevance and, consequently, promote therapeutic strategies' implementation that could impact on PFS and, more relevant, on overall survival (OS).

\section{ALK rearrangement frequency (early and late stage)}

Since the discovery of ALK translocations (mainly involving EML4 as partner gene) in NSCLC, this molecular event appeared to be a relative infrequent phenomenon, detectable in $2-7 \%$ of patients (14). Notably, almost all the dataset screened for ALK gene fusions were composed of surgically treated patients, having enough tumor tissue for comprehensive molecular analyses (15-17). In these and other surgical series ALK rearrangements are seldom identified, reporting a low frequency $(<5 \%$ in more than 300 samples), whilst percentage of positivity impressively increases looking at the advanced (stage IV) population, rising at $18 \%(18,19)$. Conversely, EGFR mutations detection remains around $15 \%$ across different disease stages (20,21). Multiple factors may justify this different behavior. A possible explanation is that ALK translocations, once associated with other genetic disrupting events, drive an aggressive metastasizing tumor phenotype, leading to its identification only at late stages of the disease. Supporting this hypothesis, also early stages ALK+ lung cancers appeared to be more spread than what initially thought: a recent retrospective analysis, performed on resected lung adenocarcinoma with clinical N0 (absence of computed tomography or positron emission tomography detectable lymph node involvement), revealed an higher percentage of pathological $\mathrm{N} 1$ or $\mathrm{N} 2$ stage (37\%) in ALK+ cases compared to other subgroups (EGFR+, KRAS+ or WT) (22). ALK+ lung cancer patients are younger, never or light smoker, diagnosed mainly on advanced stage of the disease, bearing adenocarcinoma histology with peculiar aspects: solid or sheet-like pattern, with "signet-ring" cells and invasive mucinous aspects, similar to other fusionpositive subgroups (i.e., ROS+ or RET+) (13,17-19); thus, identifying a subgroup of tumors with morphological aspects denoting itself a more aggressive phenotype $(23,24)$.

Different authors investigated the prognostic relevance of oncogene-addiction in early-stage lung cancers, but a general consensus has not been reached due to conflicting results. Identification of the right comparator group seems to be relevant: results of an European platform screening, conducted mainly in a smoker population, identifies ALK positivity (either by IHC or FISH) as predictor of better OS, whereas in other studies, evaluating never-smoker populations, the presence of ALK translocations resulted to be associated with poor disease free survival (DFS) (25-27). Similarly, other studies conducted on resected lung cancers highlight the association between ALK positivity and reduced recurrence free survival (RFS) or DFS compared to EGFR+ patients, although no differences in OS have been reported $(13,28,29)$. Multiple factors can potentially jeopardize the prognostic interpretation of ALK fusions in surgical patients: retrospective nature of these studies, heterogeneity of comparator (smokers/never-smokers, oncogenic drivers, different stages) and limited number of 
ALK+ patients. Extended follow-up, prospective and wider validation analyses, including different oncogene-addicted subgroups, could corroborate the evidence of a negative clinical impact of $A L K$ gene translocations. Nevertheless, the undoubted higher number of disease recurrences in this patients' subgroup spurs scientific community to implement peri-operative strategies aiming to a better disease control $(9,30)$.

\section{Data on ALK inhibitors in the metastatic setting}

Multiple ALK inhibitors are nowadays available in the clinical arena for advanced ALK+ lung cancer patients. Even though large clinical data are available for each compound and molecular mechanisms of resistance have been depicted, nevertheless a clear sequential strategy of administration is still missing.

Initially, crizotinib (Xalkori ${ }^{\circledR}$ ), a first generation ALK/ ROS1 inhibitor active also against c-MET, was approved by FDA in 2011 and EMA in 2012 as standard treatment for lung cancers harboring ALK fusions, identified through a companion diagnostic assay. Standard drug dosage is $250 \mathrm{mg}$ bid, based on results of randomized phase III trial Profile 1014, for both patients progressing to chemotherapy or treatment-naïve $(3,31)$.

Ceritinib (Zykadia ${ }^{\circledR}$ ) was approved by FDA in 2014 , and EMA in 2015, for ALK+ patients progressing or intolerant to crizotinib due to an improved PFS compared to chemotherapy (5.4 vs. 1.6 months) (32); moreover, based on ASCEND-4 trial's results, ceritinib got the reimbursement as first-line option (33). Initial indication was of $750 \mathrm{mg}$ once daily in fasting patients, but recent ASCEND-8 results reported equal efficacy and an improved tolerability at 450 or $600 \mathrm{mg}$ once daily with a low-fat meal (34).

Alectinib (Alecensa ${ }^{\circledR}$ ), a potent and selective ALK/ RET inhibitor, was initially approved in 2015 for patients progressing or intolerant to crizotinib therapy, based on early results of a Japanese phase I-II clinical study $(35,36)$. In 2017, phase III ALEX trial results, as above reported, pushed the alectinib approval in first-line setting, at dosage of $600 \mathrm{mg}$ bid, for ALK+ patients reporting a better toxicity profile compared to other TKI and an overwhelming PFS $(1,4)$.

Brigatinib (Alunbrig ${ }^{\circledR)}$, an ALK and mutated EGFR inhibitor, received an accelerated approval by FDA in 2017 and EMA in 2018 as second line treatment for ALK+ patients, at dosage of $90 \mathrm{mg}$ daily in a run-in phase of 7 days and after at $180 \mathrm{mg}$ daily (37). Last year have been published results of phase III ALTA-1L trial confirming a superior activity of brigatinib versus crizotinib as upfront treatment in ALK+ patients (2).

Lastly, lorlatinib (Lorbrena ${ }^{\circledR}$ ), a third generation ALKi with a high affinity for ALK and ROS-1, had recently got approval by FDA in 2018 and EMA in 2019 as third line treatment for patients who received crizotinib and at least one more compound or as second line option for patients progressing to second-generation ALKi administered upfront (38). However, in different European countries still remain available only under compassionate use. Ongoing phase III trial is evaluating the activity of this more potent compound as initial therapeutic strategy.

\section{Efforts and challenges exploring adjuvant ALK inhibitors}

If diverse efforts have been applied to understand EGFR inhibitors' role in the adjuvant setting, clinical trials in the ALK+ disease scenario are much more limited (39-41). Currently recruiting studies in adjuvant setting for ALK-rearranged patients are two phase III clinical trials: ALCHEMIST (NCT02194738) and ALINA (NCT03456076). The first one, taking advantage of wide genetic screening, enrolls stage IB $(T \geq 4 \mathrm{~cm})$-IIIA surgical treated patients after completion of standard of care treatments (chemotherapy and/or radiotherapy). ALK+ patients are randomized to receive crizotinib (at classical schedule of $250 \mathrm{mg}$ bid) versus observation, up to 24 months of treatment, in absence of disease progression or unacceptable toxicities. Other two experimental arms contemplate erlotinib for EGFR mutated patients and nivolumab for double negative subgroup. OS is the first endpoint and DFS and Safety are secondary objectives (42). The latter, ALINA, is a more recent randomized phase III trial designed to directly compare alectinib administration versus gold-standard treatment in ALK+ patients, histologically confirmed as IB ( $\mathrm{T} \geq 4 \mathrm{~cm}$ ) to IIIA stage and surgically resected at 4-12 weeks before the enrollment. Stage IIIA(N2) patients potentially candidates to radiotherapy are not included (being considered a confounding high-risk subgroup). The experimental arm requires continuous therapy with alectinib at classical schedule of $600 \mathrm{mg}$ bid for 24 months, whilst in the control arm patients receive platinum-based chemotherapy for 4 cycles. Primary endpoint is DFS and, secondary objective OS. Furthermore, alectinib's pharmacokinetic features and possible adverse events are monitored during administration. The study, started in 2018, will be terminated in 2023, with an estimated sample of 255 
patients (43).

Alectinib, even more than crizotinib, is a well-tolerated therapy with a spectrum of adverse events typical of TKI and more manageable than chemotherapy, although rare serious adverse events may happen with new-generation TKI $(1,44,45)$. Therefore, a long-course treatment in completely resected ALK+ patients is worthwhile if a real impact on long-term survival is obtained. As observed for EGFR+ lung cancer patients, initial exciting results, in terms of disease recurrence control, are impermanent and survival curves tend to converge and overlap after 4 years follow-up $(39,40)$. Consequently, major results in adjuvant approaches could be attested only by mature and complete OS data, as the ALCHEMIST trial should provide (42). Multiple ALK inhibitors are available in the clinical arena and one puzzling issue, for peri-operative management of ALK+ NSCLC, is the selection of the correct TKI to be administered, in order to gain the best clinical benefit avoiding dangerous adverse events and disease's molecular hijacking (46).

Development of genotype-directed clinical trials in the adjuvant setting is encumbered by the need of large patients recruitment, the identification of a specific targetable alteration in early-stage tumors and the timeframe of long-term follow-up, making them often less attractive. Therefore, clinical trials exploring drugs' efficacy in this setting need to be carefully planned; for instance, the ALCHEMIST trial has been designed in order to overcome these limitations (42). One major challenge is due to the identification of quite rare molecular alterations in earlystage tumors, avoiding positive patients' leak. Taking advantage of modern next-generation platforms, which give the opportunity of multi-gene analyses both at DNA and RNA level, we should consider the opportunity of screen the entire population of resected lung tumors in order to get a comprehensive molecular sub-stratification. With these data available at baseline, clinicians will be able to more easily and largely enroll patients within genotyperestricted adjuvant trials.

\section{Biological challenges of adjuvant therapy}

As we learned from experience acquired targeting ALK oncogene in the advanced stage, TKIs are able to inhibit cell proliferation, hindering tumor growth in terms of dimension and number of metastatic sites, rather than eradicate the disease. Thus, residual neoplastic cells persist and eventually become resistant to targeted therapies
$(47,48)$. Drug-sensitive tumor cells can have growth flare at therapy discontinuation or respond again at re-treatment, meaning that these cells can be curbed, but non totally killed by TKI, even of more potent-generation. This growth-suppressant property of ALKi poses a major concern about ALK targeting as adjuvant treatment, whose aim is to eliminate microscopically residual disease. It is more beneficial to treat patients with undetectable tumor cells or at evidence of macroscopic relapse? Does TKI adjuvant treatment have the same long-term survival impact than administration at disease recurrence? Reasonably, only OS data of ongoing clinical trials will answer clear-cutting to these questions, ironing out any doubts about worthiness of adjuvant therapy in oncogeneaddicted patients (49).

Neoplastic lesions are composed of heterogeneous cells population and, under pharmacological pressure imposed by TKI, clones with different biological behaviors emerge. Among them, a subpopulation of cells is able to enter in a reversible cell-cycle arrest phase becoming "dormant" or "quiescent". In this state, cancer cells not longer affected by TKI exposure, tend to acquire other genetic mutations, to undergo epigenetic modifications and to hijack tumor microenvironment eluding immune-control (50-53). Thus, persister/tolerant cells represent a reservoir of resistance mechanisms to targeted therapies and, once these take over control, tumor lesions start to grow again (48). Unfortunately, little is known about underpinning molecular events driving this "persistent" phenotype and whether ALKtargeting adjuvant therapies may cause accumulation of this cancer cells subset, altering biological history of the disease, is still to be defined. Relevant insights will be gained through clinical trials, ongoing or planned (i.e., NCT03088930 and PROMISE trials), in the neo-adjuvant setting, taking advantage of the opportunity to analyze residual tumor tissues after TKI exposure $(8,30,54)$. A deep characterization of residual tumor cells and associated tumor microenvironment represents a unique chance to deeply study TKI-induced early molecular alterations driving future resistance to targeted therapies.

Recent advances in circulating tumor DNA (ctDNA) detection and analyses are changing clinical approach for cancer patients, even in the setting of early stage NSCLC; it's possible to trace molecular evolution (i.e., identification of resistance mutations) influencing therapeutic strategies in the advance stage, follow clonal heterogeneity of cancer, help early cancer stage screening and also detect minimal residual disease (MRD) in resected patients 


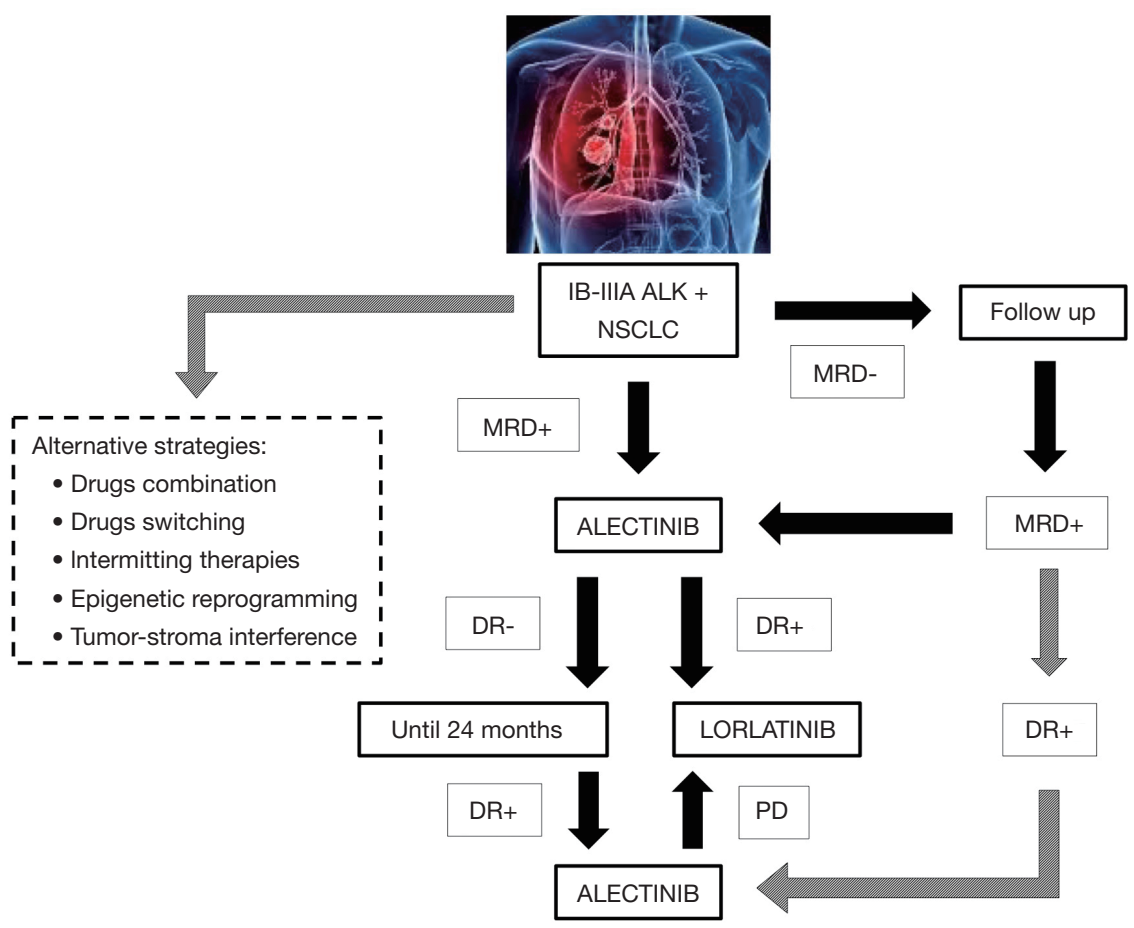

Figure 1 Hypothetic adjuvant approaches to early-stage ALK+ NSCLC. This chart shows potential therapeutic approaches to earlystage (IB-IIIA) ALK-rearranged NSCLC, not currently supported by clinical evidence. Contemplating risk stratification through MRD quantification, second and third-generation TKIs are taken into account as future key drugs, as well as other supposed strategies aimed to improve disease control rate and patients' overall survival. ALK, anaplastic lymphoma kinase; NSCLC, non-small cell lung cancer; MRD, minimal residual disease; DR, disease recurrence; PD, progressive disease; TKI, tyrosine kinase inhibitor.

(55-62). Identification of MRD has been identified as a robust marker of impending disease recurrence both in hematological and solid tumors (63-65), therefore helping in risk stratification of early stage patients (Figure 1). With new available technologies and the application of next generation sequencing on surgical specimens is possible to design patient-specific MRD panel, covering specific tumorassociated mutations rather than the entire genome (65). This implies multiple questions in the management of early stage ALK+ and other oncogene-addicted lung cancer patients: adjuvant therapy should start immediately to all patients after surgery or only at MRD positivity? Should it be monitored with blood-based targeted genomic changes and how often checked? Which is the right threshold (as absolute number or raising rate) defining patients that have to be treated? Are there specific MRD+ positive subgroups with higher risk of relapse (i.e., EML4-ALK fusion variant V3 and/or TP53 co-mutations) (66)?

The limited available experience in the adjuvant setting, mainly acquired on EGFR+ patients, is based on second- generation inhibitors and little is now about last-generation, more potent TKI and their influence on tumor's biology; data from ongoing trials will provide relevant insights $(41,43)$. Adjuvant treatments are administered for a specific timeframe (i.e., 24 months) and resistance mechanisms may not suddenly emerge. Nevertheless, drug-tolerant cell clones can proliferate and potentially hijack tumor evolution generating resistant phenotypes. Future perspectives should take in consideration also alternative strategies in order to prevent these phenomena: safe combination of drugs (given together or as switching therapies) targeting different signalling pathways; intermitting administrations which may control tumor heterogeneity and development of resistant clones; reprogramming agents (e.g., epigenetic modifiers) able to interfere with tumor cells adaptation and tumorstroma interaction $(67,68)$ (Figure 1).

The bet of adjuvant therapies with targeted agents in oncogene addicted NSCLC is to really improve chances of cure ensuring low risks for patients: minimize TKI-related adverse events and avoid molecular disruption 
of ALK-rearranged disease, preserving future therapeutic opportunities.

\section{Acknowledgments}

None.

\section{Footnote}

Conflicts of Interest: The authors have no conflicts of interest to declare.

Ethical Statement: The authors are accountable for all aspects of the work in ensuring that questions related to the accuracy or integrity of any part of the work are appropriately investigated and resolved.

\section{References}

1. Peters S, Camidge DR, Shaw AT, et al. Alectinib versus Crizotinib in Untreated ALK-Positive Non-Small-Cell Lung Cancer. N Engl J Med 2017;377:829-38.

2. Camidge DR, Kim HR, Ahn MJ, et al. Brigatinib versus Crizotinib in ALK-Positive Non-Small-Cell Lung Cancer. N Engl J Med 2018;379:2027-39.

3. Solomon BJ, Mok T, Kim DW, et al. First-Line Crizotinib versus Chemotherapy in ALK-Positive Lung Cancer N Engl J Med 2014;371:2167-77.

4. Camidge DR, Peters S, Mok T, et al. Updated efficacy and safety data from the global phase III ALEX study of alectinib (ALC) vs crizotinib (CZ) in untreated advanced ALK+ NSCLC. J Clin Oncol, 2018;36:abstr 9043.

5. Ettinger DS, Wood DE, Aisner DL, et al. NCCN guidelines insights: non-small cell lung cancer, Version 3. 2019.

6. Boyd JA, Hubbs JL, Kim DW, et al. Timing of local and distant failure in resected lung cancer: implications for reported rates of local failure. J Thorac Oncol 2010 5:211-4.

7. De Koning H, Van Der Aalst C, Ten Haaf K, et al. Effects of volume CT lung cancer screening: Mortality results of the NELSON randomised-controlled population based trial. 2018 World Conference on Lung Cancer. Abstract PL02.05. Presented September 25, 2018.

8. Zhang Y, Chen H. Neoadjuvant or adjuvant chemotherapy for non-small-cell lung cancer: Does the timing matter? J Thorac Cardiovasc Surg 2019;157:756-7.

9. Blumenthal GM, Bunn PA Jr, Chaft JE, et al. Current Status and Future Perspectives on Neoadjuvant Therapy in Lung Cancer. J Thorac Oncol 2018;13:1818-31.
10. Pignon JP, Tribodet H, Scagliotti GV, et al. Lung adjuvant cisplatin evaluation: a pooled analysis by the LACE collaborative group. J Clin Oncol 2008;26:3552-9.

11. Cancer Genome Atlas Research Network. Comprehensive molecular profiling of lung adenocarcinoma. Nature 2014;511:543-50.

12. Schram AM, Chang MT, Jonsson P, et al. Fusions in solid tumours: diagnostic strategies, targeted therapy, and acquired resistance. Nat Rev Clin Oncol 2017;14:735-48.

13. Li P, Gao Q, Jiang X, et al. Comparison of Clinicopathological Features and Prognosis between ALK Rearrangements and EGFR Mutations in Surgically Resected Early-stage Lung Adenocarcinoma. J Cancer 2019;10:61-71.

14. Soda M, Choi YL, Enomoto M, et al. Identification of the transforming EML4-ALK fusion gene in non-small-cell lung cancer. Nature 2007;448:561-6.

15. Takeuchi K, Soda M, Togashi Y, et al. RET, ROS1 and ALK fusions in lung cancer. Nat Med 2012;18:378-81.

16. Wong DW, Leung EL, So KK, et al. The EML4-ALK fusion gene is involved in various histologic types of lung cancers from nonsmokers with wild-type EGFR and KRAS. Cancer 2009;115:1723-33.

17. Pan Y, Zhang Y, Li Y, et al. ALK, ROS1 and RET fusions in 1139 lung adenocarcinomas: a comprehensive study of common and fusion pattern-specific clinicopathologic, histologic and cytologic features. Lung Cancer 2014;84:121-6.

18. Rodig SJ, Mino-Kenudson M, Dacic S, et al. Unique Clinicopathologic Features Characterize ALK-rearranged Lung Adenocarcinoma in the Western Population. Clin Cancer Res 2009;15:5216-23.

19. Shaw AT, Yeap BY, Mino-Kenudson M, et al. Clinical Features and Outcome of Patients With Non-Small-Cell Lung Cancer Who Harbor EML4-ALK. J Clin Oncol 2009;27:4247-53.

20. Paez JG, Janne PA, Lee JC, et al. EGFR mutations in lung cancer: correlation with clinical response to gefitinib therapy. Science 2004;304:1497-500.

21. Tabbò F, Nottegar A, Guerrera F, et al. Cell of origin markers identify different prognostic subgroups of lung adenocarcinoma. Hum Pathol 2018;75:167-78.

22. Seto K, Kuroda H, Yoshida T, et al. Higher frequency of occult lymph node metastasis in clinical N0 pulmonary adenocarcinoma with ALK rearrangement. Cancer Manag Res 2018;10:2117-24.

23. Warth A, Muley T, Meister M, et al. The Novel Histologic International Association for the Study of Lung Cancer/ 
American Thoracic Society/European Respiratory Society Classification System of Lung Adenocarcinoma Is a Stage-Independent Predictor of Survival. J Clin Oncol 2012;30:1438-46.

24. Hung JJ, Yeh YC, Jeng WJ, et al. Predictive Value of the International Association for the Study of Lung Cancer/ American Thoracic Society/European Respiratory Society Classification of Lung Adenocarcinoma in Tumor Recurrence and Patient Survival. J Clin Oncol 2014;32:2357-64.

25. Blackhall FH, Peters S, Bubendorf L, et al. Prevalence and Clinical Outcomes for Patients With ALK-Positive Resected Stage I to III Adenocarcinoma: Results From the European Thoracic Oncology Platform Lungscape Project. J Clin Oncol 2014;32:2780-7.

26. Kim MH, Shim HS, Kang DR, et al., Clinical and prognostic implications of ALK and ROS1 rearrangements in never-smokers with surgically resected lung adenocarcinoma. Lung Cancer 2014;83:389-95.

27. Yang P, Kulig K, Boland JM, et al., Worse diseasefree survival in never-smokers with ALK+ lung adenocarcinoma. J Thorac Oncol 2012;7:90-7.

28. Shin SH, Lee H, Jeong BH, et al. Anaplastic lymphoma kinase rearrangement in surgically resected stage IA lung adenocarcinoma. J Thorac Dis 2018;10:3460-7.

29. Chaft JE, Dagogo-Jackb I, Santini FC, et al. Clinical outcomes of patients with resected, early-stage ALKpositive lung cancer. Lung Cancer 2018;122:67-71.

30. McCoach CE, Bivona TG, Blakely CM. Neoadjuvant Oncogene-Targeted Therapy in Early Stage Non SmallCell Lung Cancer as a Strategy to Improve Clinical Outcome and Identify Early Mechanisms of Resistance. Clin Lung Cancer 2016;17:466-9.

31. Shaw AT, Kim DW, Nakagawa K, et al. Crizotinib versus chemotherapy in advanced ALK-positive lung cancer. N Engl J Med 2013;368:2385-94.

32. Crinò L, Ahn MJ, De Marinis F, et al. Multicenter Phase II Study of Whole-Body and Intracranial Activity With Ceritinib in Patients With ALK-Rearranged Non-SmallCell Lung Cancer Previously Treated With Chemotherapy and Crizotinib: Results From ASCEND-2. J Clin Oncol 2016;34:2866-73.

33. Soria JC, Tan DSW, Chiari R, et al. First-line ceritinib versus platinum-based chemotherapy in advanced ALKrearranged non-small-cell lung cancer (ASCEND-4): a randomised, open-label, phase 3 study. Lancet 2017;389:917-29.

34. Cho BC, Kim DW, Bearz A, et al. ASCEND-8: a randomized phase 1 study of ceritinib, 450 or $600 \mathrm{mg}$, taken with a low-fat meal versus $750 \mathrm{mg}$ in fasted state in patients with anaplastic lymphoma kinase (ALK)rearranged metastatic non-small cell lung cancer (NSCLC). J Thorac Oncol 2017;12:1357-67.

35. Seto T, Kiura K, Nishio M, et al. CH5424802 (RO5424802) for patients with ALK-rearranged advanced non-small-cell lung cancer (AF-001JP study): a single-arm, open-label, phase 1-2 study. Lancet Oncol 2013;14:590-8.

36. Tamura T, Kiura K, Seto T, et al. Three-year follow-up of an alectinib phase I/II study in ALK-positive non-smallcell lung cancer: AF-001JP. J Clin Oncol 2017;35:1515.

37. Kim DW, Tiseo M, Ahn MJ, et al. Brigatinib in Patients With Crizotinib-Refractory Anaplastic Lymphoma KinasePositive Non-Small-Cell Lung Cancer: A Randomized, Multicenter Phase II Trial. J Clin Oncol 2017;35:2490-8.

38. Shaw AT, Felip E, Bauer TM, et al. Lorlatinib in nonsmall-cell lung cancer with ALK or ROS1 rearrangement: an international, multicentre, open-label, single-arm firstin-man phase 1 trial. Lancet Oncol 2017;18:1590-9.

39. Zhong WZ, Wang Q, Mao WM, et al. Gefitinib versus vinorelbine plus cisplatin as adjuvant treatment for stage II-IIIA (N1-N2) EGFR-mutant NSCLC (ADJUVANT/ CTONG1104): a randomised, open-label, phase 3 study. Lancet Oncol 2018;19:139-48.

40. Pennell NA, Neal JW, Chaft JE, et al. SELECT: A Phase II Trial of Adjuvant Erlotinib in Patients With Resected Epidermal Growth Factor Receptor-Mutant Non-SmallCell Lung Cancer. J Clin Oncol 2019;37:97-104.

41. Wu YL, Herbst RS, Mann H, et al. ADAURA: Phase III, Double-blind, Randomized Study of Osimertinib Versus Placebo in EGFRMutation-positive Early-stage NSCLC After Complete Surgical Resection. Clin Lung Cancer 2018;19:e533-6.

42. NCT02194738: Genetic Testing in Screening Patients With Stage IB-IIIA Non-Small Cell Lung Cancer That Has Been or Will Be Removed by Surgery (The ALCHEMIST Screening Trial).

43. NCT03456076: A Study Comparing Adjuvant Alectinib Versus Adjuvant Platinum-Based Chemotherapy in Patients With ALK Positive Non-Small Cell Lung Cancer.

44. Pellegrino B, Facchinetti F, Bordi P, et al. Lung Toxicity in NoneSmall-Cell Lung Cancer Patients Exposed to ALK Inhibitors: Report of a Peculiar Case and Systematic Review of the Literature. Clin Lung Cancer 2018;19:e151-61.

45. Tabbò F, D'Aveni A, Tota D, et al. Pulmonary Arterial Hypertension in ALK Receptor Tyrosine Kinase-Positive 
Lung Cancer Patient: Adverse Event or Disease Spread? J Thorac Oncol 2019;14:e38-40.

46. Yoda S, Lin JJ, Lawrence MS, et al. Sequential ALK Inhibitors Can Select for Lorlatinib-Resistant Compound ALK Mutations in ALK-Positive Lung Cancer. Cancer Discov 2018;8:714-29.

47. Gainor JF, Dardaei L, Yoda S, et al. Molecular Mechanisms of Resistance to First- and Second-Generation ALK Inhibitors in ALK-Rearranged Lung Cancer. Cancer Discov 2016;6:1118-33.

48. Bivona TG, Doebele RC. A framework for understanding and targeting residual disease in oncogene-driven solid cancers. Nat Med 2016;22:472-8.

49. Ng TL, Camidge DR. Lung cancer's real adjuvant EGFR targeted therapy questions. Lancet Oncol 2018;19:15-7.

50. Sharma SV, Lee DY, Li B, et al. A chromatin-mediated reversible drug-tolerant state in cancer cell subpopulations. Cell 2010;141:69-80.

51. Bartosh TJ, Ullah M, Zeitouni S et al. Cancer cells enter dormancy after cannibalizing mesenchymal stem/stromal cells (MSCs). Proc Natl Acad Sci U S A. 2016;113:E6447-56.

52. Vallette FM, Olivier C, Lézot F, et al. Dormant, quiescent, tolerant and persister cells: Four synonyms for the same target in cancer Biochem Pharmacol 2019;162:169-76.

53. Recasens A, Munoz L. Targeting Cancer Cell Dormancy. Trends Pharmacol Sci 2019;40:128-41.

54. NCT03088930: Evaluating Crizotinib in the Neoadjuvant Setting in Patients With Non-small Cell Lung Cancer.

55. Chabon JJ, Simmons AD, Lovejoy AF, et al. Circulating tumour DNA profiling reveals heterogeneity of EGFR inhibitor resistance mechanisms in lung cancer patients. Nat Commun 2016;7:11815.

56. Murtaza M, Dawson SJ, Tsui DWY, et al. Non-invasive analysis of acquired resistance to cancer therapy by sequencing of plasma DNA. Nature 2013;497:108-12.

57. Dagogo-Jack I, Brannon AR, Ferris LA, et al. Tracking the Evolution of Resistance to ALK Tyrosine Kinase Inhibitors through Longitudinal Analysis of Circulating

Cite this article as: Tabbò F, Novello S. Expanding anaplastic lymphoma kinase therapeutic indication to early stage nonsmall cell lung cancer. Transl Lung Cancer Res 2019;8(Suppl 3):S290-S297. doi: 10.21037/tlcr.2019.07.07
Tumor DNA. JCO Precis Oncol 2018. doi: 10.1200/ PO.17.00160.

58. McCoach CE, Blakely CM, Banks KC, et al. Clinical Utility of Cell-Free DNA for the Detection of ALK Fusions and Genomic Mechanisms of ALK Inhibitor Resistance in Non-Small Cell Lung Cancer. Clin Cancer Res 2018;24:2758-70.

59. Jamal-Hanjani M, Wilson GA, McGranahan N, et al. Tracking the evolution of non-small-cell lung cancer. $\mathrm{N}$ Engl J Med 2017;376:2109-21.

60. Phallen J, Sausen M, Adleff V, et al. Direct detection of early-stage cancers using circulating tumor DNA. Sci Transl Med 2017;9. doi: 10.1126/scitranslmed.aan2415.

61. Chaudhuri AA, Chabon JJ, Lovejoy AF, et al. Early detection of molecular residual disease in localized lung cancer by circulating tumor DNA profiling. Cancer Discov 2017;7:1394-403.

62. Ivey A, Hills RK, Simpson MA, et al. Assessment of Minimal Residual Disease in Standard-Risk AML. N Engl J Med 2016;374:422-33.

63. Tie J, Wang Y, Tomasetti C, et al. Circulating tumor DNA analysis detects minimal residual disease and predicts recurrence in patients with stage II colon cancer. Sci Transl Med 2016;8:346ra92.

64. Garcia-Murillas I, Schiavon G, Weigelt B, et al. Mutation tracking in circulating tumor DNA predicts relapse in early breast cancer. Sci Transl Med 2015;7:302ra133.

65. $\mathrm{Ng} \mathrm{SB}$, Chua C, Ng M, et al. Individualised multiplexed circulating tumour DNA assays for monitoring of tumour presence in patients after colorectal cancer surgery. Sci Rep 2017;7:40737.

66. Christopoulos P, Kirchner M, Bozorgmehr F, et al. Identification of a highly lethal V3+ TP $53+$ subset in ALK+ lung adenocarcinoma. Int J Cancer 2019;144:190-9.

67. Dagogo-Jack I, Shaw AT. Tumour heterogeneity and resistance to cancer therapies. Nat Rev Clin Oncol 2018;15:81-94.

68. Chatterjee N, Bivona TG. Polytherapy and Targeted Cancer Drug Resistance. Trends Cancer 2019;5:170-82. 